\title{
Missed cancers in lung cancer screening - more than meets the eye
}

\author{
A. Devaraj
}

Received: 24 July 2014 / Accepted: 11 August 2014 / Published online: 5 September 2014

(C) European Society of Radiology 2014

\begin{abstract}
In lung cancer, early detection and diagnosis is of paramount importance. In 2011 the National Lung Screening Trial (NLST) demonstrated the effectiveness of computed tomography (CT) screening for lung cancer in reducing mortality, and results from other ongoing trials are expected to be published in the near future. A topic that has not been widely researched to date, however, is the cause for screening failure and missed lung cancers. In this issue of European Radiology, Scholten et al. describe a number of causes for false-negative screens. Some of the implications for CT screening and nodule management raised by this report are discussed.

Key Points

- Many causes exist for missed lung cancers in CT screening trials

- Endobronchial structures, the hila and mediastinum are blind spots on screening CTs

- The management of atypical nodular opacities on thoracic CT may be challenging
\end{abstract}

Keywords Lung cancer · Screening - Computed tomography · Bullous emphysema $\cdot$ Lung nodule

Missed lung cancers on chest radiographs are a well recognised source of litigation in radiology [1]. While it may be intuitive to think that most missed lung cancers on chest radiographs are due to failures of detection, previous evidence suggests that often missed lung cancers are detected but misinterpreted as benign opacities or normal structures at the time of reporting [2].

Related publication by Scholten and colleagues can be found at http://dx.doi.org/10.1007/s00330-014-3394-4.

\footnotetext{
A. Devaraj $(\bowtie)$

Department of Radiology, Royal Brompton Hospital, Sydney Street, London SW3 6NP, UK

e-mail: a.devaraj@nhs.net
}

In lung cancer screening with thoracic computed tomography (CT), there are some parallels but also dissimilarities concerning this issue. The ability of readers to detect lung nodules on CT has certainly been the subject of extensive investigation. In mainly research conditions, it has been shown that even experienced thoracic radiologists may demonstrate only moderate performance in nodule detection $[3,4]$. However, in contrast to the plain chest radiograph, much emphasis has also been placed on the issue of minimizing "overcalls" (i.e., false-positive nodules) in CT screening. The topic of false negatives in lung cancer screening has gained less attention, which is perhaps a reflection of its very high negative predictive value $(>99 \%)[5,6]$.

In this issue of European Radiology, the publication by Scholten and colleagues [7] of the NELSON trial is one of the few recent $\mathrm{CT}$ lung cancer screening studies to report on the causes of missed lung cancers. In this study, 22 missed lung cancers are described, which were visible in retrospect on the previous screening thoracic $\mathrm{CT}$. What is revealing is not that the majority of misses were due to errors of detection, but rather that in most, there was an "explanation" for the miss.

For example, in 5/22 cases, the overlooked opacity manifested as a small endobronchial lesion, in $3 / 22$ cases as mediastinal or hilar lymph nodes, and in $5 / 22$ cases as thickening of a bulla wall. Only in $2 / 22$ cases (both intra-parenchymal nodules) could no explanation be provided other than observational error.

The concept of bulla wall thickening as a marker of lung cancer is worthy of discussion. The association between lung cancer and bullae has long been recognized on plain radiographs [8], and this phenomenon has also gained attention recently in CT studies [9]. However, malignant and benign opacities in areas of emphysema demonstrate considerable overlap in appearances [10], and so, how such opacities should be managed lacks consensus.

A number of questions arise: what degree of bulla wall thickness should prompt early follow-up CT or further 
investigation? What extent of the bulla wall should be involved before malignancy is considered? Is nodular thickening of the bulla wall of more concern compared to diffuse thickening?

Further questions, currently unanswered, are also raised about the optimal management protocols in screening for other types of "atypical" opacities; for example, apical scar-like nodules (which incidentally was also identified as a cause of a missed lung cancer in this study), or para-osteophyte opacities that likely represent focal fibrosis, or endobronchial opacities that are probably mucus. Should these opacities be treated in the same manner as typical indeterminate lung nodules, and mandate follow-up CT if above a predefined size?

Analysis of large numbers of nodules from completed or ongoing lung cancer screening trials has already provided invaluable data to assist in the management of "typical" lung nodules, by identifying characteristics predictive of malignancy $[5,11]$. The National Lung Screening Trial (NLST), for example, has reported the positive predictive values for malignancy according to lung nodule size, based on an analysis of more than 7000 individuals with nodules. The same trial also reports the positive predictive value of mediastinal or hilar lymphadenopathy for malignancy (18\%) [5].

To provide similar evidence to guide the management of "atypical" opacities such as bulla wall thickening would require the analysis of large numbers of CTs demonstrating these lesions in patients without lung cancer. This is something which is currently lacking, and may not be forthcoming. Regardless, radiologists should be alert to the possibility that nodular thickening of a bulla wall may represent malignancy. The demonstration of progressive thickening on serial CTs should be sufficient to trigger pulmonologist or multidisciplinary team (MDT) referral in most cases.

It is noteworthy that in the current study by Scholten and colleagues, false-negative CT screens were due to a variety of reasons, not just observational oversight. Eight cases were due to lack of patient adherence, three due to failure of invasive workup, two due to failure of the protocol, and in 11 cases because of the rapidity of tumour development following a negative screen. In combination, these miscellaneous causes were more common than radiological error.

Just as there is no universally accepted definition for what constitutes a false positive in lung cancer screening, no such definition exists for false negatives. It should be stressed that the current study only reports on missed cancers identified after screening had finished or cancers which presented between screening rounds (interval cancers). As the authors acknowledge, equally informative data could be obtained by scrutinizing false-negative cases where cancer was diagnosed at subsequent screening rounds, but which was identifiable on a previous $\mathrm{CT}$.

Veronesi et al. [12] recently described the causes of false negatives in the COSMOS lung cancer screening study. They included within their definition of false negatives, lung cancers that were diagnosed at the time of screening which were evident as a nodule on a prior CT, but only if the lung cancer was stage II-IV (i.e., if there was a significant delay in diagnosis as demonstrated by stage progression). Interestingly, in this study [12], as in the study by Scholten et al. [7] and other previous reports [13], centrally located or endobronchial lesions are disproportionately represented in missed lung cancers.

Computer aided detection systems (CAD) have long been known to be more sensitive in nodule detection than human readers. Currently, considerable efforts are being devoted to tackle its limitations, such as improving the detection of ground glass nodules [14]. Knowledge gained from articles such as the one by Scholten et al. [7] of the characteristics of missed lung cancers may provide useful guidance for future CAD development. In the interim, it also serves to remind radiologists of their known blind spots.

Acknowledgements The scientific guarantor of this publication is Anand Devaraj. The author of this manuscript declares no relationships with any companies whose products or services may be related to the subject matter of the article. The author states that this work has not received any funding.

\section{References}

1. Baker SR, Patel RH, Yang L, Lelkes VM, Castro A III (2013) Malpractice suits in chest radiology: an evaluation of the histories of 8265 radiologists. J Thorac Imaging 28:388-391

2. Manning DJ, Ethell SC, Donovan T (2004) Detection or decision errors? Missed lung cancer from the posteroanterior chest radiograph. Br J Radiol 77:231-235

3. Armato SG III, Nitt-Gray MF, Reeves AP et al (2007) The Lung Image Database Consortium (LIDC): an evaluation of radiologist variability in the identification of lung nodules on CT scans. Acad Radiol 14:1409-1421

4. Nietert PJ, Ravenel JG, Taylor KK, Silvestri GA (2011) Influence of nodule detection software on radiologists' confidence in identifying pulmonary nodules with computed tomography. J Thorac Imaging 26:48-53

5. Church TR, Black WC, Aberle DR et al (2013) Results of initial lowdose computed tomographic screening for lung cancer. N Engl J Med 368:1980-1991

6. van Klaveren RJ, Oudkerk M, Prokop M et al (2009) Management of lung nodules detected by volume CT scanning. N Engl J Med 361: 2221-2229

7. Scholten ET, Horeweg N, de Koning HJ, Vliegenthart R, Oudkerk M, Mali WPTM et al (2014) Computed tomographic characteristics of interval and post screen carcinomas in lung cancer screening. Eur Radiol. doi:10.1007/s00330-014-3394-4

8. Tsutsui M, Araki Y, Shirakusa T, Inutsuka S (1988) Characteristic radiographic features of pulmonary carcinoma associated with large bulla. Ann Thorac Surg 46:679-683

9. Kaneda M, Tarukawa T, Watanabe F, Adachi K, Sakai T, Nakabayashi $\mathrm{H}$ (2010) Clinical features of primary lung cancer adjoining pulmonary bulla. Interact Cardiovasc Thorac Surg 10:940-944

10. Matsuoka S, Kurihara Y, Yagihashi K, Niimi H, Nakajima Y (2005) Peripheral solitary pulmonary nodule: CT findings in patients with pulmonary emphysema. Radiology 235:266-273 
11. McWilliams A, Tammemagi MC, Mayo JR et al (2013) Probability of cancer in pulmonary nodules detected on first screening CT. N Engl J Med 369:910-919

12. Veronesi G, Maisonneuve P, Spaggiari L et al (2014) Diagnostic performance of low-dose computed tomography screening for lung cancer over five years. J Thorac Oncol 9:935-939
13. Menezes RJ, Roberts HC, Paul NS et al (2010) Lung cancer screening using low-dose computed tomography in at-risk individuals: the Toronto experience. Lung Cancer 67:177-183

14. Jacobs C, van Rikxoort EM, Twellmann T et al (2014) Automatic detection of subsolid pulmonary nodules in thoracic computed tomography images. Med Image Anal 18:374-384 